GENETIC VARIATION

\title{
Linking TF binding to disease risk using pooled $\mathrm{Ch} / \mathrm{P}$-seq
}

A pooled ChIP-seq (chromatin immunoprecipitation followed by sequencing) approach for mapping quantitative trait loci (QTLs) can identify genetic variants that affect the binding of transcription factors (TFs), according to new research published in Cell. Ultimately, this approach reveals links between binding QTLs (bQTLs), chromatin architecture and complex disease at a much lower cost compared to current QTL mapping methods.

Since 2005, genome-wide association studies (GWAS) have linked thousands of genetic variants to various human phenotypes and diseases. However, the majority of associated variants are located in non-coding regions of the genome. Although many of these variants are located in known tissuespecific enhancers, whether these non-coding variants affect TF binding and gene expression remains largely unknown. Although ChIP-seq permits investigation of TF binding, the costs and human effort in performing ChIP-seq across many individuals are prohibitive, and experimental variation across experiments can make interpretation difficult.

To overcome these experimental limitations, the team pooled lymphoblastoid samples to enrich for alleles that are bound by TFs (in this case NF- $\kappa$ B, PU.1, STAT1, JunD and OCT1) with high affinity or that have trimethylated histone $\mathrm{H} 3$ lysine 4 (H3K4me3; which is associated with active transcription). The pre-ChIP and post-ChIP allele frequencies of most single-nucleotide polymorphisms (SNPs) were similar, as expected. However, the team also identified 1,533-38,944 independent bQTLs per TF - a total of $0.5-3 \%$ of all bound SNPs.

This approach also enables the investigation of cooperative binding and pioneer factors. For example, the CTCF transcription factor, which is known to be involved in transcriptional regulation and the regulation of $3 \mathrm{D}$ genome architecture, was shown to

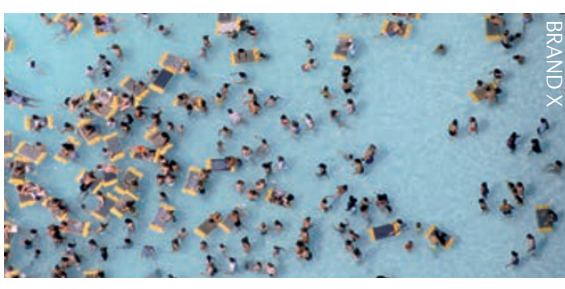

facilitate the binding of several other transcription factors. This finding indicates that CTCF is likely to be a major pioneer factor. Using Hi-C data, the team was also able to show that alleles with a higher level of TF binding (or more H3K4me3) usually have a higher number of distal contacts.

Finally, the team began to delve into the possible molecular mechanisms that link bQTLs to complex diseases, demonstrating the translational application of their approach. Importantly, any number of samples can be pooled and studied with a single ChIP-seq experiment, which should enable researchers to achieve a far deeper level of understanding of the molecular mechanisms underpinning human phenotypic variation and complex disease risk.

Bryony Jones

ORIGINAL ARTICLE Tehranchi, A. K. et al. Pooled ChIP-seq links variation in transcription factor binding to complex disease risk. Cell http://dx.doi.org/10.1016/j.cell.2016.03.041 (2016) 